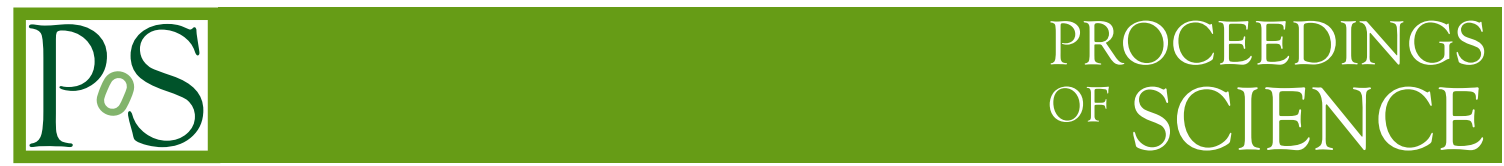

\title{
Studies of $\Lambda_{b}^{0}$ in ATLAS: decays to charmonium states and parity violating asymmetry
}

\section{Dongliang Zhang ${ }^{* \dagger}$}

University of Michigan

E-mail: zhangdon@umich.edu

The measurements of $\Lambda_{b}^{0}$ lifetime and decay asymmetry parameter in $\Lambda_{b}^{0} \rightarrow J / \psi \Lambda^{0}$ using 2011 $7 \mathrm{TeV} p p$ collision data taken by ATLAS at LHC are presented. The measured value of $\Lambda_{b}^{0}$ lifetime is $\tau_{\Lambda_{b}^{0}}=1.449 \pm 0.036$ (stat) \pm 0.017 (syst) ps, and the parity violating decay asymmetry parameter is measured to be $\alpha_{b}=0.30 \pm 0.16$ (stat) \pm 0.06 (syst).

The 15th International Conference on B-Physics at Frontier Machines at the University of Edinburgh, 14 -18 July, 2014

University of Edinburgh, $U K$

*Speaker.

On behalf of the ATLAS Collaboration 


\section{Introduction}

Although the $\Lambda_{b}^{0}$ baryon has been discovered for more than two decades, many of its basic properties are not measured as precisely as other lighter hadrons. For example, its lifetime had been a puzzle in the Tevatron days. The measurements from CDF [1] and D0 [2] have more than two standard deviation discrepancy. The CDF measurement of $\Lambda_{b}^{0}$ and $B_{d}^{0}$ lifetime ratio $R=\tau_{\Lambda_{b}^{0}} / \tau_{B_{d}^{0}}$ is also more than two standard deviation higher than the theoretical predictions. Another basic but unknown parameter before LHC is the decay asymmetry parameter of $\Lambda_{b}^{0} \rightarrow J / \psi \Lambda^{0}$. The theoretical predictions of this parameter varies in a large range, from -0.2 to 0.78 (see references in Ref. [3]). The $\Lambda_{b}^{0} \rightarrow J / \psi\left(\mu^{+} \mu^{-}\right) \Lambda^{0}\left(p \pi^{-}\right)$events in 2011 collision data collected by ATLAS detector [4] at LHC are used to measure the lifetime and the decay asymmetry parameter.

\section{2. $\Lambda_{b}^{0}$ reconstruction}

Data taken by ATLAS detector in 2011 using single muon, dimuon and $J / \psi$ muon trigger corresponding to an integrated luminosity of about $5 \mathrm{fb}^{-1}$ are used to reconstruct $\Lambda_{b}^{0}$. In the preselection, $J / \psi$ candidates are reconstructed from muon pairs, and $\Lambda^{0}$ candidates are reconstructed by fitting two inner detector tracks. Both $J / \psi$ and $\Lambda^{0}$ candidates are required to be within a mass window around their mass values [5]. The four tracks are then fitted simultaneously using the $\Lambda_{b}^{0} \rightarrow J / \psi\left(\mu^{+} \mu^{-}\right) \Lambda^{0}\left(p \pi^{-}\right)$hypothesis, in which the masses of $J / \psi$ and $\Lambda^{0}$ are fixed to their values. Good fit quality $\chi^{2} / N_{\text {dof }}<3$ is required. Furthermore, the refitted $\Lambda^{0}$ is required to have $p_{\mathrm{T}}>3.5 \mathrm{GeV}$ and transverse decay length $L_{x y}>10 \mathrm{~mm}$ with respect to the dimuon vertex to suppress combinatorial backgrounds. To reject $B_{d}^{0}$ background, a $B_{d}^{0} \rightarrow J / \psi\left(\mu^{+} \mu^{-}\right) K_{S}^{0}\left(\pi^{+} \pi^{-}\right)$ hypothesis fit is also performed to the track-quadruplet and the event will be kept only if the cumulative probability for $\Lambda_{b}^{0}$ is higher than the $B_{d}^{0}$ hypothesis by 0.05 . The reconstructed mass of $\Lambda_{b}^{0}$ and $\bar{\Lambda}_{b}^{0}$ candidates are shown in Fig. 1 . The $\Lambda_{b}^{0}$ and $\bar{\Lambda}_{b}^{0}$ events have similar distributions, and they are combined for these measurements.

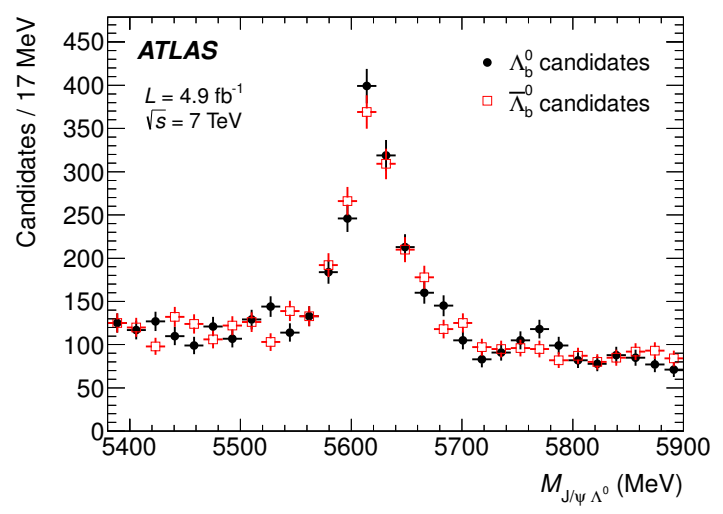

Figure 1: The mass distribution of the reconstructed $\Lambda_{b}^{0}$ and $\bar{\Lambda}_{b}^{0}$ candidates [6].

\section{3. $\Lambda_{b}^{0}$ lifetime measurement}

The lifetime of $\Lambda_{b}^{0}$ are measured by a two dimensional simultaneous fit of the reconstructed 
mass and proper decay time of each selected candidate [6]. The mass of $\Lambda_{b}^{0}$ is also measured from this fit.

The unbinned likelihood function is given by

$$
L=\prod_{i=1}^{N}\left[f_{\mathrm{sig}} \mathscr{M}_{\mathrm{s}}\left(m_{i} \mid \boldsymbol{\delta}_{m i}\right) \mathscr{T}_{\mathrm{s}}\left(\tau_{i} \mid \boldsymbol{\delta}_{\tau i}\right) w_{\mathrm{s}}\left(\boldsymbol{\delta}_{m i}, \boldsymbol{\delta}_{\tau i}\right)+\left(1-f_{\mathrm{sig}}\right) \mathscr{M}_{\mathrm{b}}\left(m_{i} \mid \boldsymbol{\delta}_{m i}\right) \mathscr{T}_{\mathrm{b}}\left(\tau_{i} \mid \boldsymbol{\delta}_{\tau i}\right) w_{\mathrm{b}}\left(\boldsymbol{\delta}_{m i}, \boldsymbol{\delta}_{\tau i}\right)\right]
$$

where $f_{\text {sig }}$ is the fraction of signal events; $m_{i}$ and $\tau_{i}$ are the reconstructed mass and proper decay time of the $i$ th event. The reconstructed mass distribution and proper decay time distribution are found uncorrelated, so they can be modeled separately. For the reconstructed mass, the probability density function (PDF) for signal $\left(\mathscr{M}_{\mathrm{s}}\left(m_{i} \mid \delta_{m i}\right)\right)$ is modeled using Gaussian distribution, and for background $\left(\mathscr{M}_{\mathrm{b}}\left(m_{i} \mid \delta_{m i}\right)\right)$, a linear distribution is used. The proper decay time PDF of signal events, $\mathscr{T}_{\mathrm{s}}\left(\tau_{i} \mid \delta_{\tau i}\right)$, is given by a exponential function. For the proper decay time PDF of background, $\mathscr{T}_{\mathrm{b}}\left(\tau_{i} \mid \delta_{\tau i}\right)$, the prompt background is modeled using a delta function and a symmetric exponential function, and non-prompt contribution are modeled by two exponential functions. All these proper decay time PDFs are convolved by a Gaussian resolution function. The distribution of reconstructed mass uncertainty and proper decay time uncertainties for signal and background, $w_{\mathrm{s}}\left(\boldsymbol{\delta}_{m i}, \boldsymbol{\delta}_{\tau i}\right)$ and $w_{\mathrm{b}}\left(\boldsymbol{\delta}_{m i}, \boldsymbol{\delta}_{\tau i}\right)$, are extracted from data and have the same form. The values of mass and lifetime measurement from the fit is shown in Fig. 2. The PDF is projected to the reconstructed mass and proper decay time distributions and is compared with data. The major systematic un-
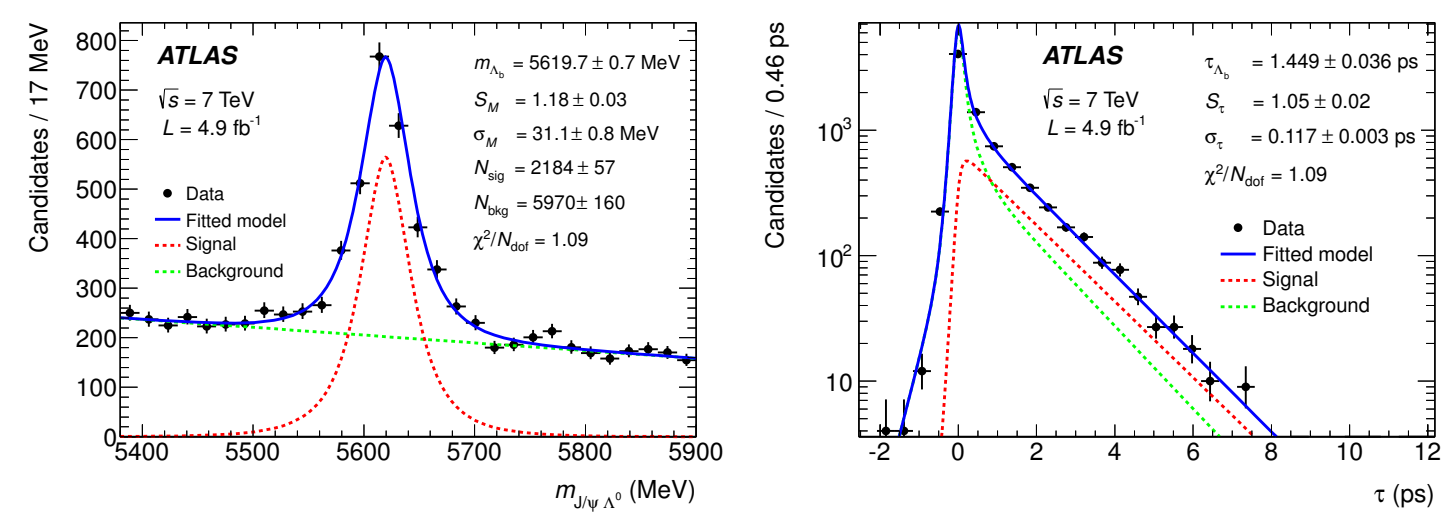

Figure 2: Mass-proper decay time fit results projected to mass (left) and proper decay time (right) and compared with data [6].

certainties are related to the $\Lambda^{0}$ reconstruction and selection performed at trigger level. The final measured values of the mass and lifetime

$$
\begin{aligned}
m_{\Lambda_{b}^{0}} & =5619.7 \pm 0.7(\text { stat }) \pm 1.1(\text { syst }) \mathrm{MeV} \\
\tau_{\Lambda_{b}^{0}} & =1.449 \pm 0.036(\text { stat }) \pm 0.017(\text { syst }) \mathrm{ps}
\end{aligned}
$$

and the ratio,

$$
R=\tau_{\Lambda_{b}^{0}} / \tau_{B_{d}^{0}}^{\mathrm{PDG}}=0.960 \pm 0.025 \text { (stat) } \pm 0.016 \text { (syst) }
$$

are consistent with the later results from CMS [7] and $\mathrm{LHCb}$ [8]. But the recent lifetime measurement from CDF [9] is still more than two standard deviation higher than this value. 


\section{4. $\alpha_{b}$ measurement}

Taking $\lambda_{\Lambda}$ and $\lambda_{J / \psi}$ to represent the helicity of the $\Lambda^{0}$ and the $J / \psi$, the decay $\Lambda_{b}^{0} \rightarrow J / \psi \Lambda^{0}$ can be described by four helicity amplitudes $A\left(\lambda_{\Lambda}, \lambda_{J / \psi}\right): a_{+} \equiv A(1 / 2,0), a_{-} \equiv A(-1 / 2,0), b_{+} \equiv$ $A(-1 / 2,-1)$ and $b_{-} \equiv A(1 / 2,1)$. The distribution of the five decay angles shown in Fig. $3, \Omega=$ $\left(\theta, \theta_{1}, \phi_{1}, \theta_{2}, \phi_{2}\right)$, depends on these helicity parameters, and can be written as [10]:

$$
w(\Omega)=\frac{1}{(4 \pi)^{3}} \sum_{i} f_{1 i}(\vec{A}) f_{2 i}\left(P, \alpha_{\Lambda}\right) F_{i}(\Omega) .
$$
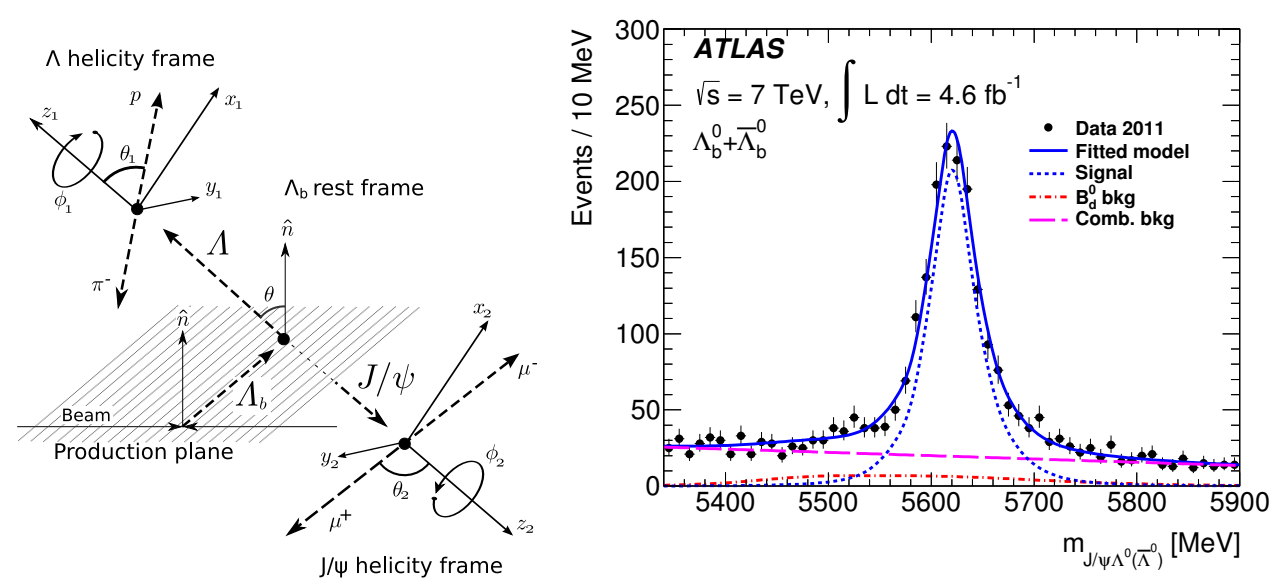

Figure 3: Definition of the decay angles (left) and the reconstructed mass of the selected $\Lambda_{b}^{0}$ and $\bar{\Lambda}_{b}^{0}$ candidates (right) [3].

As the overall polarization is 0 due to the symmetry of the detector, the PDF is simplified and only six out of twenty terms remain, for $i=0,2,4,6,18$, and 19. Five unconstrained parameters can be determined from data, and they are chosen as: the decay asymmetry parameter $\alpha_{b}=\left|a_{+}\right|^{2}-$ $\left|a_{-}\right|^{2}+\left|b_{+}\right|^{2}-\left|b_{-}\right|^{2}$, two helicity amplitude ratio parameters $\left(k_{+}=\frac{\left|a_{+}\right|}{\sqrt{\left|a_{+}\right|^{2}+\left|b_{+}\right|^{2}}}, k_{-}=\frac{\left|b_{-}\right|}{\sqrt{\left|a_{-}\right|^{2}+\left|b_{-}\right|^{2}}}\right)$ and two relative phase parameters $\left(\Delta_{+}, \Delta_{-}\right)$. Table 1 shows the form of $f_{1 i}$ and $F_{i}$ of these six terms. The parameter $f_{2 i}$ equals the value of the decay asymmetry parameter of $\Lambda^{0} \rightarrow p \pi^{-}, \alpha_{\Lambda}=$ $0.642 \pm 0.013$ [5], for $i=2,6,18,19$, and is unit for $i=0$ or 4 .

Table 1: The coefficients $f_{1 i}$, and $F_{i}$ of the remaining six terms of the simplified PDF [3].

\begin{tabular}{|c|c|c|}
\hline$i$ & $f_{1 i}$ & $F_{i}$ \\
\hline 0 & 1 & 1 \\
\hline 2 & $\left(k_{+}^{2}+k_{-}^{2}-1\right)+\alpha_{b}\left(k_{+}^{2}-k_{-}^{2}\right)$ & $\cos \theta_{1}$ \\
\hline 4 & $\frac{1}{4}\left[\left(3 k_{-}^{2}-3 k_{+}^{2}-1\right)+3 \alpha_{b}\left(1-k_{-}^{2}-k_{+}^{2}\right)\right]$ & $\frac{1}{2}\left(3 \cos ^{2} \theta_{2}-1\right)$ \\
\hline 6 & $-\frac{1}{4}\left[\left(k_{+}^{2}+k_{-}^{2}-1\right)+\alpha_{b}\left(3+k_{+}^{2}-k_{-}^{2}\right)\right]$ & $\frac{1}{2}\left(3 \cos ^{2} \theta_{2}-1\right) \cos \theta_{1}$ \\
\hline 18 & $\frac{3}{\sqrt{2}}\left[\frac{1-\alpha_{b}}{2} \sqrt{k_{-}^{2}\left(1-k_{-}^{2}\right)} \cos \left(-\Delta_{-}\right)-\frac{1+\alpha_{b}}{2} \sqrt{k_{+}^{2}\left(1-k_{+}^{2}\right)} \cos \left(\Delta_{+}\right)\right]$ & $\sin \theta_{1} \sin \theta_{2} \cos \theta_{2} \cos \left(\phi_{1}+\phi_{2}\right)$ \\
\hline 19 & $-\frac{3}{\sqrt{2}}\left[\frac{1-\alpha_{b}}{2} \sqrt{k_{-}^{2}\left(1-k_{-}^{2}\right)} \sin \left(-\Delta_{-}\right)-\frac{1+\alpha_{b}}{2} \sqrt{k_{+}^{2}\left(1-k_{+}^{2}\right)} \sin \left(\Delta_{+}\right)\right]$ & $\sin \theta_{1} \sin \theta_{2} \cos \theta_{2} \sin \left(\phi_{1}+\phi_{2}\right)$ \\
\hline
\end{tabular}

To determine the value of the helicity parameters, the following function of the helicity parameters,

$$
\chi^{2}=\sum_{i} \sum_{j}\left(\left\langle F_{i}\right\rangle^{\text {expected }}-\left\langle F_{i}\right\rangle\right) V_{i j}^{-1}\left(\left\langle F_{j}\right\rangle^{\text {expected }}-\left\langle F_{j}\right\rangle\right),
$$


is minimized, where $\left\langle F_{i}\right\rangle^{\text {expected }}$ is the expected value of $F_{i}$ predicted by the given helicity parameters; $\left\langle F_{i}\right\rangle$ and $V_{i j}$ are the measured average values and their covariance matrix elements [3].

The expected values are calculated from the PDF (Eqn. 4.2) and corrected for the detector effects, which is estimated from Monte Carlo (MC) simulation. Fig. 4 shows the expected distributions of $\cos \theta_{1}$ and $\cos \theta_{2}$ for various $\alpha_{b}$ values.
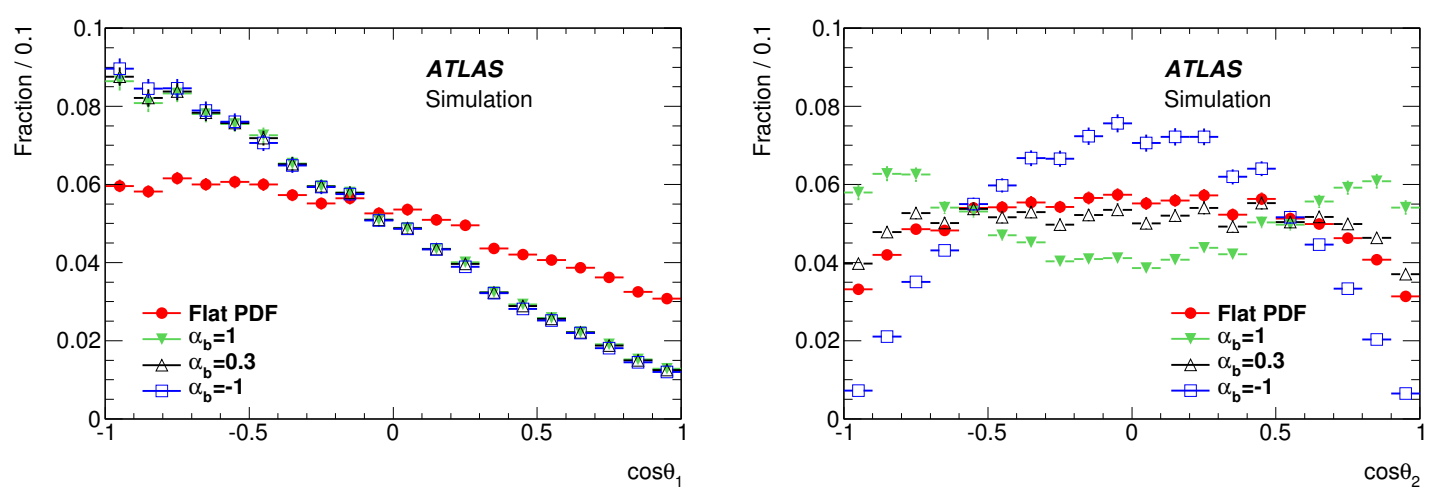

Figure 4: $\cos \theta_{1}$ and $\cos \theta_{2}$ distribution of MC events for various $\alpha_{b}$ value settings. In the flat PDF, $\alpha_{b}=0$ and other parameters are set so that the distributions are flat at generator level. For $\alpha_{b}=-1,0.3$ and 1 , other parameters are set to $k_{+}=0.21$ and $k_{-}=0.13$ (measured values), and $\Delta_{+}=\Delta_{-}=0$ [3].

To further reduce the combinatorial background, proper decay time $\tau>3.5 \mathrm{ps}$ is required, and the events are selected requiring cumulative probability for $\Lambda_{b}^{0}$ larger than the one for $B_{d}^{0}$ hypothesis. The reconstructed mass distribution of the selected events is shown in Fig. 3. The combinatorial background is estimated from sidebands. Both data and $\mathrm{MC}$ are used to estimate the $B_{d}^{0}$ background.

The final result of the helicity amplitude measurement is

$$
\begin{aligned}
& \alpha_{b}=0.30 \pm 0.16(\text { stat }) \pm 0.06 \text { (syst) } \\
& k_{+}=0.21_{-0.21}^{+0.14}(\text { stat }) \pm 0.13 \text { (syst) } \\
& k_{-}=0.13_{-0.13}^{+0.20}(\text { stat }) \pm 0.15 \text { (syst) }
\end{aligned}
$$

corresponding to the magnitude of helicity amplitudes

$$
\begin{array}{lll}
\left|a_{+}\right|=0.17_{-0.17}^{+0.12}(\text { stat }) \pm 0.09 \text { (syst) }, & & \left|a_{-}\right|=0.59_{-0.07}^{+0.06}(\text { stat }) \pm 0.03 \text { (syst) } \\
\left|b_{+}\right|=0.79_{-0.05}^{+0.04}(\text { stat }) \pm 0.02 \text { (syst) }, & & \left|b_{-}\right|=0.08_{-0.08}^{+0.13}(\text { stat }) \pm 0.06 \text { (syst) } .
\end{array}
$$

The phase parameters $\left(\Delta_{+}\right.$and $\left.\Delta_{-}\right)$are consistent with the entire allowed range. The systematic uncertainty mainly come from combinatorial background shape estimation and MC statistics.

The $\Lambda_{b}^{0}$ decay has large amplitudes $\left|a_{-}\right|$and $\left|b_{+}\right|$, which means the negative-helicity states for $\Lambda^{0}$ are preferred and the $\Lambda^{0}$ and $J / \psi$ from $\Lambda_{b}^{0}$ decay are highly polarized. Adding in quadrature the statistical and systematic uncertainties, the observed value of $\alpha_{b}$ is consistent with the recent measurement $\alpha_{b}=0.05 \pm 0.17$ (stat) \pm 0.07 (syst) by LHCb [11] at the level of one standard deviation. However, it is not consistent with the expectation from pQCD [12] $\left(\alpha_{b}\right.$ in the range from -0.17 to -0.14$)$, and HQET [13] $\left(\alpha_{b}=0.78\right)$ at a level of about 2.6 and 2.8 standard deviations, respectively. 


\section{Summary}

The $\Lambda_{b}^{0} \rightarrow J / \psi\left(\mu^{+} \mu^{-}\right) \Lambda^{0}\left(p \pi^{-}\right)$events in ATLAS $7 \mathrm{TeV}$ collision data taken in 2011 are used to measure the $\Lambda_{b}^{0}$ lifetime and decay helicity amplitudes. $\Lambda_{b}^{0}$ lifetime is measured to be $\tau_{\Lambda_{b}^{0}}=$ $1.449 \pm 0.036$ (stat) \pm 0.017 (syst) ps, consistent with the world average and the later measurements by other experiments, and the parity violating decay asymmetry parameter $\alpha_{b}$ is measured to be $\alpha_{b}=0.30 \pm 0.16$ (stat) \pm 0.06 (syst), compatible with the LHCb results [11] but lying in the middle of two theory predictions, both separated by more than two standard deviations. The $\Lambda^{0}$ from $\Lambda_{b}^{0}$ decay is found highly polarized.

\section{References}

[1] The CDF Collaboration, T. Aaltonen et al., Measurement of b hadron lifetimes in exclusive decays containing a $J / \psi$ in p $\bar{p}$ collisions at $\sqrt{s}=1.96 \mathrm{TeV}$, Phys. Rev. Lett. 106 (2011) 121804, [hep-ex/1012.3138].

[2] The D0 Collaboration, V. M. Abazov et al., Measurement of the $\Lambda_{b}^{0}$ lifetime in the exclusive decay $\Lambda_{b}^{0} \rightarrow J / \psi \Lambda^{0}$ in p p collisions at $\sqrt{s}=1.96 \mathrm{TeV}$, Phys. Rev. D 85 (2012) 112003, [hep-ex/1204.2340].

[3] ATLAS Collaboration, Measurement of the parity-violating asymmetry parameter $\alpha_{b}$ and the helicity amplitudes for the decay $\Lambda_{b} \rightarrow J / \psi+\Lambda$ with the ATLAS detector, Phys. Rev. D 89 (2014) 092009, [hep-ex/1404.1071].

[4] ATLAS Collaboration, The ATLAS Experiment at the CERN Large Hadron Collider, JINST 3 (2008) S08003.

[5] Particle Data Group, K. Olive et al., Review of Particle Physics, Chin. Phys. C 38 (2014) 090001.

[6] ATLAS Collaboration, Measurement of the $\Lambda_{b}$ lifetime and mass in the ATLAS experiment, Phys. Rev. D 87 (2013) 032002, [hep-ex/1207.2284].

[7] CMS Collaboration, S. Chatrchyan et al., Measurement of the $\Lambda_{b}^{0}$ lifetime in pp collisions at $\sqrt{s}=7$ $\mathrm{TeV}$, JHEP 07 (2013) 163, [hep-ex/1304 . 7495] .

[8] The LHCb collaboration, R. Aaij et al., Precision measurement of the $\Lambda_{b}^{0}$ baryon lifetime, Phys. Rev. Lett. 111 (2013) 102003, [hep-ex/1307.2476].

[9] The CDF Collaboration, T. A. Aaltonen et al., Mass and lifetime measurements of bottom and charm baryons in p $\bar{p}$ collisions at $\sqrt{s}=1.96$ TeV, Phys. Rev. D 89 (2014) 072014, [hep-ex/1403.8126].

[10] J. Hrivnac, R. Lednicky, and M. Smizanska, Feasibility of beauty baryon polarization measurement in $\Lambda^{0} J / \psi$ decay channel by ATLAS LHC, J. Phys. G 21 (1995) 629-638, [hep-ph/9405231] .

[11] The LHCb Collaboration, R. Aaij et al., Measurements of the $\Lambda_{b}^{0} \rightarrow J / \psi \Lambda$ decay amplitudes and the $\Lambda_{b}^{0}$ polarisation in pp collisions at $\sqrt{s}=7 \mathrm{TeV}$, Phys. Lett. B 724 (2013) 27, [hep-ex/1302.5578].

[12] C.-H. Chou, H.-H. Shih, S.-C. Lee, and H.-n. Li, $\Lambda_{b} \rightarrow \Lambda J / \psi$ decay in perturbative QCD, Phys. Rev. D 65 (2002) 074030, [hep-ph/0112145].

[13] Z. Ajaltouni, E. Conte, and O. Leitner, $\Lambda_{b}$ decays into $\Lambda$-vector, Phys. Lett. B 614 (2005) 165-173, [hep-ph/0412116]. 